\title{
RESTRICTION OF CHARACTERS TO SUBGROUPS OF WREATH PRODUCTS AND BASIC SETS FOR THE SYMMETRIC GROUP
}

\author{
JEAN-BAPTISTE GRAMAIN AND ADRIANA MARCIUK
}

\begin{abstract}
In this paper, we give the decomposition into irreducible characters of the restriction to the wreath product $\mathbb{Z}_{p-1} \prec \mathfrak{S}_{w}$ of any irreducible character of $\left(\mathbb{Z}_{p} \rtimes \mathbb{Z}_{p-1}\right)$ < $\mathfrak{S}_{w}$, where $p$ is any odd prime, $w \geq 0$ is an integer, and $\mathbb{Z}_{p}$ and $\mathbb{Z}_{p-1}$ denote the cyclic groups of order $p$ and $p-1$ respectively. This answers the question of how to decompose the restrictions to $p$-regular elements of irreducible characters of the symmetric group $\mathfrak{S}_{n}$ in the $\mathbb{Z}$-basis corresponding to the $p$-basic set of $\mathfrak{S}_{n}$ described by Brunat and Gramain in [1]. The result is given in terms of the Littlewood-Richardson coefficients for the symmetric group.
\end{abstract}

\section{INTRODUCTION}

Let $G$ be a finite group and $\operatorname{Irr}(G)$ be the set of irreducible complex characters of $G$. Let $p$ be a prime (dividing $|G|$ ), and let $\mathcal{C}$ be the set of $p$-regular elements of $G$. For each $\chi \in \mathbb{C} \operatorname{Irr}(G)$, we define a class function $\chi^{\mathcal{C}}$ of $G$ by letting

$$
\chi^{\mathcal{C}}(g)= \begin{cases}\chi(g) & \text { if } g \in \mathcal{C} \\ 0 & \text { otherwise. }\end{cases}
$$

One of the fundamental results of Brauer's Theory is the existence of a surjective homomorphism, called the decomposition homomorphism,

$$
d:\left\{\begin{array}{rl}
\mathbb{Z} \operatorname{Irr}(G) & \longrightarrow \mathbb{Z} \operatorname{IBr}_{p}(G) \\
\chi & \longmapsto \chi^{\mathcal{C}}
\end{array},\right.
$$

where $\operatorname{IBr}_{p}(G)$ is the set of irreducible ( $p$-modular) Brauer characters of $G$. The matrix $D$ of $d$ in the bases $\operatorname{Irr}(G)$ and $\operatorname{IBr}_{p}(G)$ is the (p-modular) decomposition matrix of $G$. Up to reordering the rows and columns, the matrix $D$ is diagonal by blocks, which gives partitions of $\operatorname{Irr}(G)$ and $\operatorname{IBr}_{p}(G)$ into $p$-blocks.

While finding the decomposition matrix of a group is a very difficult problem, basic sets can sometimes help computing Brauer characters and/or the decomposition matrix $D$, or at least reduce the problem. We call $p$-basic set for $G$ any subset $\mathcal{B} \subset \operatorname{Irr}(G)$ such that the family $\mathcal{B}^{\mathcal{C}}=\left\{\chi^{\mathcal{C}}, \chi \in \mathcal{B}\right\}$ is a $\mathbb{Z}$-basis for the $\mathbb{Z}$-module generated by $\operatorname{Irr}^{\mathcal{C}}(G)=\left\{\chi^{\mathcal{C}}, \chi \in \operatorname{Irr}(G)\right\}$. In particular, $|\mathcal{B}|$ is the number of $p$ regular conjugacy classes of $G$. One can also define the notion of $p$-basic set for a $p$-block of $G$, and one shows easily that, if each $p$-block $b$ of $G$ has a $p$-basic set $\mathcal{B}_{b}$, then the union of the $\mathcal{B}_{b}$ 's is a $p$-basic set for $G$.

If $\mathcal{B}$ is a $p$-basic set for $G$, and if we write $\chi^{\mathcal{C}}=\sum_{\psi \in \mathcal{B}} n_{\chi \psi} \cdot \psi^{\mathcal{C}}\left(\chi \in \operatorname{Irr}(G), n_{\chi \psi} \in \mathbb{Z}\right)$ and $N_{\mathcal{B}}=\left(n_{\chi \psi}\right)_{\chi \in \operatorname{Irr}(G), \psi \in \mathcal{B}}$, and $D_{\mathcal{B}}$ for the (square) sub-matrix of $D$ whose rows 
correspond to $\mathcal{B}$, then we have $D=N_{\mathcal{B}} D_{\mathcal{B}}$, so that computing the matrix $N_{\mathcal{B}}$ reduces the problem of finding $D$ to computing (the smaller matrix) $D_{\mathcal{B}}$.

In [1], the authors describe, for any integer $n$ and odd prime $p$, a $p$-basic set $\mathcal{B}$ for the symmetric group $\mathfrak{S}_{n}$. The object of the present paper is, in this case, to describe completely the matrix $N_{\mathcal{B}}$. It should be noted that another $p$-basic set for $\mathfrak{S}_{n}$ was previously known (see $[3$, Section 6.3$]$ ), but that $\mathcal{B}$ has further properties which allow it to restrict to a $p$-basic set for the alternating group $\mathcal{A}_{n}$.

Throughout this paper, we let $n \geq 1$ be any integer, and $p$ be an odd prime. The irreducible complex characters of the symmetric group $\mathfrak{S}_{n}$ are canonically labelled by partitions of $n$, and we write $\operatorname{Irr}\left(\mathfrak{S}_{n}\right)=\left\{\chi_{\lambda} \mid \lambda \vdash n\right\}$. For any $\lambda \vdash n$, we write $n=|\lambda|$, the size of $\lambda$. The distribution of irreducible characters of $\mathfrak{S}_{n}$ into $p$ blocks is described by the Nakayama Conjecture (see [3, 6.1.21]). Each partition $\lambda$ of $n$ is completely and uniquely determined by its $p$-core $\gamma_{p}(\lambda)$ and its $p$-quotient $q_{p}(\lambda)$. The $p$-core $\gamma_{p}(\lambda)$ is the partition, of some integer $s$, obtained by removing from $\lambda$ all the hooks of length divisible by $p$, and the $p$-quotient $q_{p}(\lambda)$ is a $p$ tuple $\left(\lambda^{1}, \lambda^{2}, \ldots, \lambda^{p}\right)$ of partitions whose sizes add up to the integer $w$ (written $\left.\left(\lambda^{1}, \lambda^{2}, \ldots, \lambda^{p}\right) \Vdash w\right)$, called the $p$-weight of $\lambda$, and such that $n=s+p w$. Then two characters $\chi_{\lambda}, \chi_{\mu} \in \operatorname{Irr}\left(\mathfrak{S}_{n}\right)$ belong to the same $p$-block of $\mathfrak{S}_{n}$ if and only if $\gamma_{p}(\lambda)=\gamma_{p}(\mu)$. In particular, if that is the case, then $\lambda$ and $\mu$ have the same $p$ weight, and characters in the $p$-block of $\mathfrak{S}_{n}$ corresponding to the $p$-core $\gamma \vdash s$ are labelled by the $p$-tuples of partitions $\left(\nu^{1}, \nu^{2}, \ldots, \nu^{p}\right) \Vdash w$, where $w=(n-s) / p$, and the $p$-block is said to have $p$-weight $w$.

In [1], the authors show that $\left\{\chi_{\lambda} \in \operatorname{Irr}\left(\mathfrak{S}_{n}\right) \mid q_{p}(\lambda)=\left(\lambda^{1}, \ldots, \lambda^{p}\right)\right.$ with $\left.\lambda^{r}=\emptyset\right\}$ is a $p$-basic set for $\mathfrak{S}_{n}$, where $r=\frac{p+1}{2}$. To prove this, they construct, for each $w \geq 0$, a generalized perfect isometry between the set of irreducible characters of a $p$-block $b$ of $p$-weight $w$ and the set of irreducible characters of the wreath product $\left(\mathbb{Z}_{p} \rtimes \mathbb{Z}_{p-1}\right)$ 乙 $\mathfrak{S}_{w}$, where $\mathbb{Z}_{p}$ and $\mathbb{Z}_{p-1}$ denote the cyclic groups of order $p$ and $p-1$ respectively. The irreducible characters of $\left(\mathbb{Z}_{p} \rtimes \mathbb{Z}_{p-1}\right) \curlywedge \mathfrak{S}_{w}$ can be parametrized by the $p$-tuples $\nu=\left(\nu^{1}, \ldots, \nu^{p}\right) \Vdash w$ of partitions of $w$ (see Section 3), in such a way that a character has the subgroup $\mathbb{Z}_{p}^{w}$ of $\left(\mathbb{Z}_{p} \rtimes \mathbb{Z}_{p-1}\right) \curlywedge \mathfrak{S}_{w}=\mathbb{Z}_{p}^{w} \rtimes\left(\mathbb{Z}_{p-1} \prec \mathfrak{S}_{w}\right)$ in its kernel if and only if it is labelled by $\nu=\left(\nu^{1}, \ldots, \nu^{p}\right) \Vdash w$ such that $\nu^{r}=\emptyset$. We write $\operatorname{Irr}\left(\left(\mathbb{Z}_{p} \rtimes \mathbb{Z}_{p-1}\right) \curlywedge \mathfrak{S}_{w}\right)=\left\{\chi^{\nu} \mid \nu \Vdash w\right\}$. We also let $\mathcal{B}_{b}=\left\{\chi_{\lambda} \in \operatorname{Irr}(b) \mid q_{p}(\lambda)=\right.$ $\left(\lambda^{1}, \ldots, \lambda^{p}\right)$ with $\left.\lambda^{r}=\emptyset\right\}$ be the $p$-basic set for $b$ constructed in [1]. The results of [1] show that there is an explicit bijection $\chi_{\lambda} \longmapsto \chi_{\tilde{\lambda}}$ from $\operatorname{Irr}(b)$ to itself, which restricts to the identity on $\mathcal{B}_{b}$, as well as explicitly determined signs $\left\{\varepsilon(\lambda), \chi_{\lambda} \in\right.$ $\operatorname{Irr}(b)\}$ such that, if we write $\chi_{\lambda}^{\mathcal{C}}=\sum_{\chi_{\mu} \in \mathcal{B}_{b}} n_{\lambda \mu} \cdot \chi_{\mu}^{\mathcal{C}}\left(\chi_{\lambda} \in \operatorname{Irr}(b), n_{\lambda \mu} \in \mathbb{Z}\right)$, then the coefficients $n_{\lambda \mu}\left(\chi_{\lambda} \in \operatorname{Irr}(b), \chi_{\mu} \in \mathcal{B}_{b}\right)$ are given by

$$
n_{\lambda \mu}=\varepsilon(\lambda) \varepsilon(\mu)\left\langle\operatorname{Res}_{\mathbb{Z}_{p-1} \rtimes \mathfrak{S}_{w}}^{\left(\mathbb{Z}_{p} \mathbb{Z}_{p-1}\right) \imath \mathfrak{S}_{w}}\left(\chi^{q_{p}(\tilde{\lambda})}\right), \operatorname{Res}_{\mathbb{Z}_{p-1} \rtimes \mathfrak{S}_{w}}^{\left(\mathbb{Z}_{p} \rtimes \mathbb{Z}_{p-1}\right) \mathfrak{S}_{w}}\left(\chi^{q_{p}(\tilde{\mu})}\right)\right\rangle_{\mathbb{Z}_{p-1} \imath \mathfrak{S}_{w}} .
$$

Note that, by construction, for any $\chi_{\mu} \in \mathcal{B}_{b}$, we have $q_{p}(\tilde{\mu})=\left(\nu^{1}, \ldots, \nu^{p}\right)$ with $\nu^{r}=\emptyset$. Thus $\chi^{q_{p}(\tilde{\mu})}$ has $\mathbb{Z}_{p}^{w}$ in its kernel, and $\operatorname{Res}_{\mathbb{Z}_{p-1} \backslash \mathfrak{S}_{w}}^{\left(\mathbb{Z}_{w}\right) 2 \mathfrak{S}_{w}}\left(\chi^{q_{p}(\tilde{\mu})}\right)$ is actually an irreducible character of $\mathbb{Z}_{p-1} \prec \mathfrak{S}_{w}$. Hence, in order to decompose any restriction to $p$-regular elements of an irreducible character of $\mathfrak{S}_{n}$ as a $\mathbb{Z}$-linear combination of the restrictions of characters in the basic set $\mathcal{B}$, it is sufficient to compute the decomposition into irreducible characters of $\mathbb{Z}_{p-1} \prec \mathfrak{S}_{w}$ of any irreducible character of $\left(\mathbb{Z}_{p} \rtimes \mathbb{Z}_{p-1}\right) \mathcal{} \mathfrak{S}_{w}$. This decomposition is given by our main result, Theorem 5.1. 
The paper is organised as follows. In Section 2, we recall classical results about the conjugacy classes and irreducible complex characters of wreath products. These results are then applied to the groups $\left(\mathbb{Z}_{p} \rtimes \mathbb{Z}_{p-1}\right) \succ \mathfrak{S}_{w}$ and $\mathbb{Z}_{p-1} \prec \mathfrak{S}_{w}$ in Section 3 , and the irreducible characters of these groups are parametrized in ways that are compatible (see Theorem 3.3). In Section 4 , we describe the characters of $\left(\mathbb{Z}_{p} \rtimes\right.$

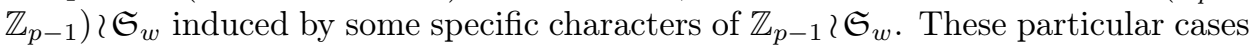
form the basis for the computations of Section 5 , where we explicitly decompose into irreducibles the induction to $\left(\mathbb{Z}_{p} \rtimes \mathbb{Z}_{p-1}\right) \prec \mathfrak{S}_{w}$ of any irreducible character of $\mathbb{Z}_{p-1} \prec \mathfrak{S}_{w}$ (see Theorem 5.1). This in turn provides a formula for any of the scalar products appearing in Equation (1).

\section{Conjugacy Classes And IRREDucible CharaCters of Wreath PROduCtS}

Throughout this section, we let $N$ be a finite group and $w \geq 1$ be an integer, and consider the wreath product $N \backslash \mathfrak{S}_{w}$. That is, $N \backslash \mathfrak{S}_{w}$ is the semidirect product $N^{w} \rtimes \mathfrak{S}_{w}$, where $\mathfrak{S}_{w}$ acts by permutation on the $w$ copies of $N$. For a complete description of wreath products and their representations, we refer to [3, Chapter 4].

Let $s$ be the number of conjugacy classes of $N$, and let $g_{1}, \ldots, g_{s}$ be representatives for the conjugacy classes of $N$. Then the conjugacy classes of $N$ ᄀ $\mathfrak{S}_{w}$ can be parametrized by the $s$-tuples of partitions of $w$ as follows. The elements of $N<\mathfrak{S}_{w}$ are of the form $(h ; \sigma)=\left(\left(h_{1}, \ldots, h_{w}\right) ; \sigma\right)$, with $h_{1}, \ldots, h_{w} \in N$ and $\sigma \in \mathfrak{S}_{w}$. For any such element, write $\sigma=\sigma_{1} * \cdots * \sigma_{c(\sigma)}$, a product of disjoint cycles. Then, for any $1 \leq \nu \leq c(\sigma)$, we have $\sigma_{\nu}=\left(j_{\nu}, j_{\nu} \sigma_{\nu}, \ldots, j_{\nu} \sigma_{\nu}^{k_{\nu}-1}\right)$ (where $\sigma_{\nu}$ is a $k_{\nu}$-cycle), and we define the $\nu$-th cycle product of $(h ; \sigma)$ by

$$
g_{\nu}(h ; \sigma)=h_{j_{\nu}} \cdot h_{j_{\nu} \sigma_{\nu}^{-1}} \cdot h_{j_{\nu} \sigma_{\nu}^{-2}} \cdots \cdot h_{j_{\nu} \sigma_{\nu}^{-\left(k_{\nu}-1\right)}} .
$$

In particular, $g_{\nu}(h ; \sigma) \in N$. We then form $s$ partitions $\left(\pi_{1}, \ldots, \pi_{s}\right)$ as follows: each $1 \leq \nu \leq c(\sigma)$ gives a cycle of length $k_{\nu}$ in $\pi_{i}$ if the cycle product $g_{\nu}(h ; \sigma)$ is conjugate to $g_{i}$ in $N$. The resulting $s$-tuple of partitions of $w$ describes the cycle structure of $(h ; \sigma)$, and two elements of $N \imath \mathfrak{S}_{w}$ are conjugate if and only if they have the same cycle structure.

The irreducible complex characters of $N<\mathfrak{S}_{w}$ can also be parametrized by the $s$-tuples of partitions of $w$ as follows. Let $\operatorname{Irr}(N)=\left\{\omega_{1}, \ldots, \omega_{s}\right\}$. Take any $\alpha=$ $\left(\alpha^{1}, \ldots, \alpha^{s}\right) \Vdash w$ and consider the irreducible character $\prod_{i=1}^{s} \omega_{i}^{\left|\alpha^{i}\right|}$ of the base group $N^{w}$. It can be extended in a natural way to its inertia subgroup $N \imath \mathfrak{S}_{\left|\alpha^{1}\right|} \times$ $\cdots \times N<\mathfrak{S}_{\left|\alpha^{s}\right|}$, giving the irreducible character $\prod_{i=1}^{s} \widetilde{\omega_{i}^{\left|\alpha^{i}\right|}}$. For each $1 \leq i \leq s$, the irreducible character $\widetilde{\omega_{i}^{\left|\alpha^{i}\right|}}$ of $N<\mathfrak{S}_{\left|\alpha^{i}\right|}$ is given as follows: if $(f ; \pi) \in N<\mathfrak{S}_{\left|\alpha^{i}\right|}$ has cycle products $g_{\nu}(f ; \pi)(1 \leq \nu \leq c(\pi))$, then $\widetilde{\omega_{i}^{\left|\alpha^{i}\right|}}(f ; \pi)=\prod_{\nu=1}^{c(\pi)} \omega_{i}\left(g_{\nu}(f ; \pi)\right)$ (see [3, Lemma 4.3.9]). Any extension of $\prod_{i=1}^{s} \omega_{i}^{\left|\alpha^{i}\right|}$ to $N\left\langle\mathfrak{S}_{\left|\alpha^{1}\right|} \times \cdots \times N \backslash \mathfrak{S}_{\left|\alpha^{s}\right|}\right.$ is of the form $\Omega^{\alpha}=\prod_{i=1}^{s}\left(\widetilde{\omega_{i}^{\left|\alpha^{i}\right|}} \otimes \Upsilon_{\alpha_{i}}\right)$, where, for each $1 \leq i \leq s, \Upsilon_{\alpha_{i}} \in \mathbb{C} \operatorname{Irr}\left(N 乙 \mathfrak{S}_{\left|\alpha^{i}\right|}\right)$ is defined by $\Upsilon_{\alpha_{i}}(f ; \pi)=\chi_{\alpha_{i}}(f ; \pi)$ for all $(f ; \pi) \in N\left\langle\mathfrak{S}_{\left|\alpha^{i}\right|}\right.$ (and $\chi_{\alpha_{i}} \in \operatorname{Irr}\left(\mathfrak{S}_{\left|\alpha^{i}\right|}\right)$, see $[3,4.3 .15])$. Then $\aleph^{\alpha}:=\operatorname{Ind}_{\prod_{i=1}^{s} N l \mathfrak{S}_{\left|\alpha^{i}\right|}}^{s}\left(\Omega^{\alpha}\right) \in \operatorname{Irr}\left(N<\mathfrak{S}_{w}\right)$. Different $\alpha \Vdash w$ give different irreducible characters of $N \imath \mathfrak{S}_{w}$, and any irreducible character of $N \imath \mathfrak{S}_{w}$ can be obtained in this way (see [3, Theorem 4.3.34]). 


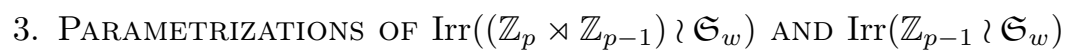

From now on, and throughout the paper, we fix an odd prime $p$, and we let $r=\frac{p+1}{2}$. We write $I$ for the set $\{1,2, \ldots, p\} \backslash\{r\}$. We let $H=\mathbb{Z}_{p-1}$ and $G=\mathbb{Z}_{p} \rtimes \mathbb{Z}_{p-1}$, and, for any integer $k \geq 1$, we let $H_{k}=H \imath \mathfrak{S}_{k}$ and $G_{k}=G \imath \mathfrak{S}_{k}$.

We start by describing the irreducible characters of $G$ and their restrictions to $H$. The irreducible complex characters of $G$ are described as follows. We have $\operatorname{Irr}(G)=\left\{\psi_{1}, \ldots, \psi_{p}\right\}$, with $\psi_{i}(1)=1$ for $i \in I$, and $\psi_{r}(1)=p-1$. More precisely, writing $\eta_{1}=1_{\mathbb{Z}_{p}}$ for the trivial character of $\mathbb{Z}_{p}$, we have

$$
\operatorname{Ind}_{\mathbb{Z}_{p}}^{G}\left(1_{\mathbb{Z}_{p}}\right)=\sum_{i \in I} \psi_{i} \text { and } \operatorname{Res}_{\mathbb{Z}_{p}}^{G}\left(\psi_{i}\right)=1_{\mathbb{Z}_{p}}(i \in I),
$$

and

$$
\operatorname{Res}_{\mathbb{Z}_{p}}^{G}\left(\psi_{r}\right)=\eta_{2}+\cdots+\eta_{p} \text { and } \operatorname{Ind}_{\mathbb{Z}_{p}}^{G}\left(\eta_{i}\right)=\psi_{r}(2 \leq i \leq p),
$$

where $\left\{\eta_{2}, \ldots, \eta_{p}\right\}=\operatorname{Irr}\left(\mathbb{Z}_{p}\right) \backslash\left\{1_{\mathbb{Z}_{p}}\right\}$.

Now write $\operatorname{Irr}(H)=\left\{\theta_{i} \mid i \in I\right\}=\left\{\theta_{1}=1_{H}, \theta_{2}, \ldots, \theta_{r-1}, \theta_{r+1}, \ldots, \theta_{p}\right\}$. For any $i \in I, \psi_{i}$ has $\mathbb{Z}_{p} \triangleleft G$ in its kernel. Thus, without loss of generality, we can choose the labelling such that $\psi_{i}=\theta_{i} \circ \varpi$, where $\varpi: G=\mathbb{Z}_{p} \rtimes \mathbb{Z}_{p-1} \longrightarrow \mathbb{Z}_{p-1}=H$ is the canonical surjection. In particular,

$$
\operatorname{Res}_{H}^{G}\left(\psi_{i}\right)=\theta_{i} \quad \text { for all } i \in I .
$$

To describe $\operatorname{Res}_{H}^{G}\left(\psi_{r}\right)$, we start by noticing that $\psi_{r}(g)=0$ for all $g \in G \backslash \mathbb{Z}_{p}$. Indeed, by the first orthogonality relation, we have

$$
\sum_{g \in \mathbb{Z}_{p}} \psi_{r}(g) \overline{\psi_{r}(g)}=\sum_{2 \leq i, j \leq p} \sum_{g \in \mathbb{Z}_{p}} \eta_{i}(g) \overline{\eta_{j}(g)}=\sum_{2 \leq i \leq p} \sum_{g \in \mathbb{Z}_{p}} \eta_{i}(g) \overline{\eta_{i}(g)}=p(p-1) .
$$

Since also $\sum_{g \in G} \psi_{r}(g) \overline{\psi_{r}(g)}=|G|=p(p-1)$, this yields

$$
\sum_{g \in G \backslash \mathbb{Z}_{p}} \psi_{r}(g) \overline{\psi_{r}(g)}=\sum_{g \in G \backslash \mathbb{Z}_{p}}\left|\psi_{r}(g)\right|^{2}=0,
$$

so that $\psi_{r}(g)=0$ for all $g \in G \backslash \mathbb{Z}_{p}$. Since $\psi_{r}(1)=p-1$, this gives

$$
\operatorname{Res}_{H}^{G}\left(\psi_{r}\right)=(p-1) \delta_{1},
$$

where $\delta_{1}$ is the indicator function of (the conjugacy class in $H$ of) 1 . Now, by the second orthogonality relation, we have, for any $h \in H$

$$
\sum_{i \in I} \theta_{i}(h)=\sum_{i \in I} \theta_{i}(h) \theta_{i}(1)=\left|C_{H}(1)\right| \delta_{1}(h)=(p-1) \delta_{1}(h) .
$$

Hence

$$
\operatorname{Res}_{H}^{G}\left(\psi_{r}\right)=\sum_{i \in I} \theta_{i}
$$

If we now take any integer $w \geq 1$, then the irreducible complex characters of $G_{w}$ and $H_{w}$ are constructed as in Section 2.

The irreducible complex characters of $G_{w}=G>\mathfrak{S}_{w}$ are parametrized by the $p$-tuples of partitions of $w$ as follows. For any $\alpha=\left(\alpha^{1}, \ldots, \alpha^{p}\right) \Vdash w, \chi^{\alpha} \in \operatorname{Irr}\left(G_{w}\right)$ is given 
by

$$
\chi^{\alpha}=\operatorname{Ind}_{\prod_{1 \leq i \leq p} G_{\left|\alpha^{i}\right|}}^{G_{w}}\left(\Psi^{\alpha}\right)=\operatorname{Ind}_{\prod_{1 \leq i \leq p}^{G_{w}} G_{\left|\alpha^{i}\right|}}\left(\prod_{1 \leq i \leq p} \widetilde{\psi_{i}^{\left|\alpha^{i}\right|}} \otimes \varphi_{\alpha^{i}}\right),
$$

where, for any $1 \leq i \leq p$ and $(f ; \pi) \in G_{\left|\alpha^{i}\right|}$ with cycle products $g_{\nu}(f ; \pi)(1 \leq \nu \leq$ $c(\pi))$, we have $\widetilde{\psi_{i}^{\left|\alpha^{i}\right|}}(f ; \pi)=\prod_{\nu=1}^{c(\pi)} \psi_{i}\left(g_{\nu}(f ; \pi)\right)$ and $\varphi_{\alpha^{i}}(f ; \pi)=\chi_{\alpha^{i}}(\pi)$.

Note for future reference that, in the above notation, if $\alpha^{r}=\emptyset$, then $\mathbb{Z}_{p}^{w} \subseteq \operatorname{ker}\left(\chi^{\alpha}\right)$. Indeed, for all $1 \leq i \leq p, i \neq r$, we have $\operatorname{Res}_{\mathbb{Z}_{p}}^{G}\left(\psi_{i}\right)=1_{\mathbb{Z}_{p}} ;$ thus $\operatorname{Res}_{\mathbb{Z}_{p}^{\sigma^{\alpha i} \mid}}^{G^{\left|\alpha^{i}\right|}}\left(\psi_{i}^{\left|\alpha^{i}\right|}\right)=$ $1_{\mathbb{Z}_{p}^{\left|\alpha^{i}\right|}}$, so that $\widetilde{\psi_{i}^{\left|\alpha^{i}\right|}}(g)=\widetilde{\psi_{i}^{\left|\alpha^{i}\right|}}(1)$ for all $g \in \mathbb{Z}_{p}^{\left|\alpha^{i}\right|}$, and $\left.\widetilde{\left(\psi_{i}^{\left|\alpha^{i}\right|}\right.} \otimes \varphi_{\alpha_{i}}\right)(g)=\widetilde{\left(\psi_{i}^{\left|\alpha^{i}\right|}\right.} \otimes$ $\left.\varphi_{\alpha_{i}}\right)(1)$ for all $g \in \mathbb{Z}_{p}^{\left|\alpha^{i}\right|}$. Since $\mathbb{Z}_{p}^{w} \leq G^{w} \triangleleft G \succ \mathfrak{S}_{w}$, we easily get that, for all $g \in \mathbb{Z}_{p}^{w}$, $\chi^{\alpha}(g)=\chi^{\alpha}(1)$. In particular, if $\alpha^{r}=\emptyset$, then $\chi^{\alpha}=\xi \circ \bar{\varpi}$ for some $\xi \in \operatorname{Irr}\left(H_{w}\right)$, where $\bar{\varpi}: G_{w}=\left(\mathbb{Z}_{p}\right)^{w} \rtimes\left(\mathbb{Z}_{p-1} \imath \mathfrak{S}_{w}\right) \longrightarrow \mathbb{Z}_{p-1} \imath \mathfrak{S}_{w}=H_{w}$ is the canonical surjection.

The irreducible complex characters of $H_{w}=H \imath \mathfrak{S}_{w}$ are parametrized by the $(p-1)$ tuples of partitions of $w$ as follows. For any $\alpha=\left(\alpha^{1}, \ldots, \alpha^{r-1}, \alpha^{r+1}, \ldots, \alpha^{p}\right) \Vdash w$, $\xi^{\alpha} \in \operatorname{Irr}\left(H_{w}\right)$ is given by

$$
\xi^{\alpha}=\operatorname{Ind}_{\prod_{i \in I} H_{\left|\alpha^{i}\right|}}^{H_{w}}\left(\Theta^{\alpha}\right)=\operatorname{Ind}_{\prod_{i \in I} H_{\left|\alpha^{i}\right|}}^{H_{w}}\left(\prod_{i \in I} \widetilde{\theta_{i}^{\left|\alpha^{i}\right|}} \otimes \zeta_{\alpha^{i}}\right),
$$

where, for any $i \in I$ and $(f ; \pi) \in H_{\left|\alpha^{i}\right|}$ with cycle products $g_{\nu}(f ; \pi)(1 \leq \nu \leq c(\pi))$, we have $\widetilde{\theta_{i}^{\left|\alpha^{i}\right|}}(f ; \pi)=\prod_{\nu=1}^{c(\pi)} \theta_{i}\left(g_{\nu}(f ; \pi)\right)$ and $\zeta_{\alpha^{i}}(f ; \pi)=\chi_{\alpha^{i}}(\pi)$.

The following result will be useful when we next consider the restriction to $H_{w}$ of some irreducible characters of $G_{w}$.

Lemma 3.1. For any integer $k \geq 1, i \in I$ and $\lambda \vdash k$, we have

$$
\operatorname{Res}_{H_{k}}^{G_{k}}\left(\widetilde{\psi_{i}^{k}}\right)=\widetilde{\theta_{i}^{k}} \quad \text { and } \quad \operatorname{Res}_{H_{k}}^{G_{k}}\left(\varphi_{\lambda}\right)=\zeta_{\lambda} .
$$

Proof. Take any $(f ; \pi) \in H_{k}$ (i.e. $(f ; \pi)=(1,(f ; \pi)) \in \mathbb{Z}_{p}^{k} \rtimes H_{k}=G_{k}$, where $f \in H^{k}$ and $\left.\pi \in \mathfrak{S}_{k}\right)$, and let $g_{\nu}(f ; \pi)(1 \leq \nu \leq c(\pi))$ be the cycle products of $(f ; \pi)$. Note that, for all $1 \leq \nu \leq c(\pi), g_{\nu}(f ; \pi) \in H$ (as a product of elements of $H)$. Since, by $(2), \operatorname{Res}_{H}^{G}\left(\psi_{i}\right)=\theta_{i}$, this yields

$$
\widetilde{\psi_{i}^{k}}(f ; \pi)=\prod_{\nu=1}^{c(\pi)} \psi_{i}\left(g_{\nu}(f ; \pi)\right)=\prod_{\nu=1}^{c(\pi)} \theta_{i}\left(g_{\nu}(f ; \pi)\right)=\widetilde{\theta_{i}^{k}}(f ; \pi),
$$

as claimed. The second part is immediate, as, for any $(f ; \pi) \in H_{k}$, we have $\varphi_{\lambda}(f ; \pi)=\chi_{\lambda}(\pi)=\zeta_{\lambda}(f ; \pi)$.

We will also need the following in Section 4 .

Lemma 3.2. For any integer $k \geq 1$ and any $(f ; \pi) \in H_{k}$ with cycle products $g_{\nu}(f ; \pi)(1 \leq \nu \leq c(\pi))$, we have

$$
\widetilde{\psi_{r}^{k}}(f ; \pi)=\left\{\begin{array}{cl}
(p-1)^{c(\pi)} & \text { if } g_{\nu}(f ; \pi)=1 \text { for all } 1 \leq \nu \leq c(\pi) \\
0 & \text { otherwise. }
\end{array}\right.
$$


Proof. Take any $(f ; \pi) \in H_{k}$ as in the proof of Lemma 3.1 and again note that, for all $1 \leq \nu \leq c(\pi), g_{\nu}(f ; \pi) \in H$. We therefore have

$$
\widetilde{\psi_{r}^{k}}(f ; \pi)=\prod_{\nu=1}^{c(\pi)} \psi_{r}\left(g_{\nu}(f ; \pi)\right)=\prod_{\nu=1}^{c(\pi)} \operatorname{Res}_{H}^{G}\left(\psi_{r}\right)\left(g_{\nu}(f ; \pi)\right) .
$$

The result immediately follows from (3).

We can now show how our parametrizations for $\operatorname{Irr}\left(G_{w}\right)$ and $\operatorname{Irr}\left(H_{w}\right)$ are related.

Theorem 3.3. Take any $\left(\alpha^{1}, \ldots, \alpha^{r-1}, \emptyset, \alpha^{r+1}, \ldots \alpha^{p}\right) \Vdash w$.

If we let $\alpha=\left(\alpha^{1}, \ldots, \alpha^{r-1}, \alpha^{r+1}, \ldots \alpha^{p}\right)$ and $\hat{\alpha}=\left(\alpha^{1}, \ldots, \alpha^{r-1}, \emptyset, \alpha^{r+1}, \ldots \alpha^{p}\right)$, then we have

$$
\operatorname{Res}_{H_{w}}^{G_{w}}\left(\chi^{\hat{\alpha}}\right)=\xi^{\alpha} .
$$

Proof. Let $\alpha=\left(\alpha^{1}, \ldots, \alpha^{r-1}, \alpha^{r+1}, \ldots \alpha^{p}\right)$ and $\hat{\alpha}=\left(\alpha^{1}, \ldots, \alpha^{r-1}, \emptyset, \alpha^{r+1}, \ldots \alpha^{p}\right)$ be as above. Then

$$
\chi^{\hat{\alpha}}=\operatorname{Ind}_{\prod_{1 \leq i \leq p} G_{\left|\alpha^{i}\right|}}^{G_{w}}\left(\Psi^{\hat{\alpha}}\right)=\operatorname{Ind}_{\prod_{i \in I}^{G_{w}} G_{\left|\alpha^{i}\right|}}\left(\Psi^{\alpha}\right),
$$

where $\Psi^{\alpha}=\prod_{i \in I} \widetilde{\psi_{i}^{\left|\alpha^{i}\right|}} \otimes \varphi_{\alpha^{i}}$

Let $\sigma_{1}, \ldots, \sigma_{m}$ be left coset representatives for the Young subgroup $\prod_{i \in I} \mathfrak{S}_{\left|\alpha^{i}\right|}$ of $\mathfrak{S}_{w}$. In particular, $\left(1 ; \sigma_{1}\right), \ldots,\left(1 ; \sigma_{m}\right)$ are also left coset representatives for $\prod_{i \in I} H_{\left|\alpha^{i}\right|}$ in $H_{w}$, and for $\prod_{i \in I} G_{\left|\alpha^{i}\right|}$ in $G_{w}$ (where, in the first instance, $\left(1 ; \sigma_{i}\right)=$ $\left(1_{H} ; \sigma_{i}\right)$ for $1 \leq i \leq m$ and, in the second, $\left(1 ; \sigma_{i}\right)=\left(1_{G} ; \sigma_{i}\right)$ for $\left.1 \leq i \leq m\right)$. Since it will always be clear which group we are working in, we will denote all these coset representatives as $\sigma_{1}, \ldots, \sigma_{m}$.

By the formula for character induction, for all $g \in H_{w}$, we have

$$
\chi^{\hat{\alpha}}(g)=\sum_{k=1}^{m} \overbrace{\Psi^{\alpha}}\left(\sigma_{k} g \sigma_{k}^{-1}\right),
$$

where

$$
\overbrace{\Psi^{\alpha}}\left(\sigma_{k} g \sigma_{k}^{-1}\right)=\left\{\begin{array}{ccc}
0 & \text { if } & \sigma_{k} g \sigma_{k}^{-1} \notin \prod_{i \in I} G_{\left|\alpha^{i}\right|}, \\
\Psi^{\alpha}\left(\sigma_{k} g \sigma_{k}^{-1}\right) & \text { if } & \sigma_{k} g \sigma_{k}^{-1} \in \prod_{i \in I} G_{\left|\alpha^{i}\right|} .
\end{array}\right.
$$

Now, if $g=(f ; \rho) \in G_{w}$ (with $\left.f \in H^{w} \leq G^{w}\right)$, then, for all $1 \leq k \leq m$, we have (see $[3,4.2 .6])$,

$$
\sigma_{k} g \sigma_{k}^{-1}=\left(1 ; \sigma_{k}\right)(f ; \rho)\left(1 ; \sigma_{k}\right)^{-1}=\left(f_{\sigma_{k}} ; \sigma_{k} \rho \sigma_{k}^{-1}\right),
$$

where, if $f=\left(\left(1, f_{1}\right),\left(1, f_{2}\right), \ldots,\left(1, f_{w}\right)\right) \in G^{w}$ (with $\left.\left(f_{1}, \ldots, f_{w}\right) \in H^{w}\right)$, then $f_{\sigma_{k}}=\left(\left(1, f_{\sigma_{k}^{-1}(1)}\right),\left(1, f_{\sigma_{k}^{-1}(2)}\right), \ldots,\left(1, f_{\sigma_{k}^{-1}(w)}\right)\right)$. In particular, $f_{\sigma_{k}} \in H^{w}$, and $\sigma_{k} g \sigma_{k}^{-1}=\left(f_{\sigma_{k}} ; \sigma_{k} \rho \sigma_{k}^{-1}\right) \in H_{w}$. Hence, for all $1 \leq k \leq m$, we have $\sigma_{k} g \sigma_{k}^{-1} \in$ $\prod_{i \in I} G_{\left|\alpha^{i}\right|}$ if and only if $\sigma_{k} g \sigma_{k}^{-1} \in \prod_{i \in I} H_{\left|\alpha^{i}\right|}$. If that is the case, then we can write $\sigma_{k} g \sigma_{k}^{-1}=\prod_{i \in I}\left(g_{i} ; \rho_{i}\right)$, where $\left(g_{i} ; \rho_{i}\right) \in H_{\left|\alpha^{i}\right|}$ for all $i \in I$, and, still in that case, 
we obtain

$$
\begin{aligned}
\Psi^{\alpha}\left(\sigma_{k} g \sigma_{k}^{-1}\right) & \left.=\prod_{i \in I} \widetilde{\left[\psi_{i}^{\left|\alpha^{i}\right|}\right.} \otimes \varphi_{\alpha^{i}}\right]\left(g_{i} ; \rho_{i}\right) \\
& =\prod_{i \in I} \widetilde{\psi_{i}^{\left|\alpha^{i}\right|}}\left(g_{i} ; \rho_{i}\right) \cdot \varphi_{\alpha^{i}}\left(g_{i} ; \rho_{i}\right) \\
& =\prod_{i \in I} \widetilde{\operatorname{Res}_{H_{\left|\alpha^{i}\right|} \mid}^{G^{i}}}\left(\widetilde{\psi_{i}^{\left|\alpha^{i}\right|}}\right)\left(g_{i} ; \rho_{i}\right) \cdot \operatorname{Res}_{H_{\left|\alpha^{i}\right|} \mid}^{G_{\alpha^{i} \mid}}\left(\varphi_{\alpha^{i}}\right)\left(g_{i} ; \rho_{i}\right) \\
& =\prod_{i \in I} \theta_{i}^{\left|\alpha^{i}\right|}\left(g_{i} ; \rho_{i}\right) \cdot \zeta_{\alpha^{i}}\left(g_{i} ; \rho_{i}\right) \quad \text { (by Lemma 3.1) } \\
& =\prod_{i \in I}\left[\widetilde{\theta_{i}^{\left|\alpha^{i}\right|}} \otimes \zeta_{\alpha^{i}}\right]\left(g_{i} ; \rho_{i}\right) \\
& =\left[\prod_{i \in I} \widetilde{\theta_{i}^{\left|\alpha^{i}\right|}} \otimes \zeta_{\alpha^{i}}\right]\left(\sigma_{k} g \sigma_{k}^{-1}\right) \\
& =\Theta^{\alpha}\left(\sigma_{k} g \sigma_{k}^{-1}\right) .
\end{aligned}
$$

We therefore have, for any $1 \leq k \leq m$,

$$
\overbrace{\Psi^{\alpha}}\left(\sigma_{k} g \sigma_{k}^{-1}\right)=\left\{\begin{array}{ccc}
0 & \text { if } & \sigma_{k} g \sigma_{k}^{-1} \notin \prod_{i \in I} H_{\left|\alpha^{i}\right|}, \\
\Theta^{\alpha}\left(\sigma_{k} g \sigma_{k}^{-1}\right) & \text { if } & \sigma_{k} g \sigma_{k}^{-1} \in \prod_{i \in I} H_{\left|\alpha^{i}\right|} .
\end{array}\right.
$$

Since $\sigma_{1}, \ldots, \sigma_{m}$ are also left coset representatives for $\prod_{i \in I} H_{\left|\alpha^{i}\right|}$ in $H_{w}$, we get, for all $g \in H_{w}$,

$$
\chi^{\hat{\alpha}}(g)=\sum_{k=1}^{m} \overbrace{\Psi^{\alpha}}\left(\sigma_{k} g \sigma_{k}^{-1}\right)=\sum_{k=1}^{m} \overbrace{\Theta^{\alpha}}\left(\sigma_{k} g \sigma_{k}^{-1}\right)=\operatorname{Ind}_{\prod_{i \in I}^{H_{w}} H_{\left|\alpha^{i}\right|}}\left(\Theta^{\alpha}\right)(g)=\xi^{\alpha}(g),
$$

as claimed.

Remark 3.4. In view of the observation we made when parametrizing the irreducible characters of $G_{w}$, the statement of Theorem 3.3 can be rephrased as: if $\chi \in \operatorname{Irr}\left(G_{w}\right)$ is labelled by $\left(\alpha^{1}, \ldots, \alpha^{r-1}, \emptyset, \alpha^{r+1}, \ldots \alpha^{p}\right)$, then $\chi=\xi \circ \bar{\varpi}$, where $\xi \in \operatorname{Irr}\left(H_{w}\right)$ is labelled by $\left(\alpha^{1}, \ldots, \alpha^{r-1}, \alpha^{r+1}, \ldots \alpha^{p}\right)$ and $\bar{\varpi}: G_{w} \longrightarrow H_{w}$ is the canonical surjection.

\section{INDUCTION OF SOME SPECIAL CHARACTERS}

In this section, we fix any integer $k \geq 1$, and we will describe the induced characters $\operatorname{Ind}_{H_{k}}^{G_{k}}\left(\widetilde{\theta_{i}^{k}} \otimes \zeta_{\alpha}\right)$ for $i \in I$ and $\alpha \vdash k$ (see Theorem 4.5). We start by some results on multiplicities.

Lemma 4.1. For any $i \in I$, the multiplicity of the irreducible character $\widetilde{\theta_{i}^{k}}$ in $\operatorname{Res}_{H_{k}}^{G_{k}}\left(\widetilde{\psi_{r}^{k}}\right)$ is 1 . 
Proof. Take any $i \in I$, and let $A_{i, r}$ be the multiplicity of $\widetilde{\theta_{i}^{k}}$ in $\operatorname{Res}_{H_{k}}^{G_{k}}\left(\widetilde{\psi_{r}^{k}}\right)$. Then

$$
\begin{aligned}
A_{i, r} & =\frac{1}{\left|H_{k}\right|} \sum_{(f ; \pi) \in H_{k}} \widetilde{\psi_{r}^{k}}(f ; \pi) \cdot \widetilde{\widetilde{\theta_{i}^{k}}(f ; \pi)} \\
& =\frac{1}{\left|H_{k}\right|} \sum_{\pi \in \mathfrak{S}_{k}} \sum_{\substack{f \in H^{k} \text { such that } \\
g_{\nu}(f ; \pi)=1 \text { for all } 1 \leq \nu \leq c(\pi)}}(p-1)^{c(\pi)} \cdot \widetilde{\widetilde{\theta}_{i}^{k}(f ; \pi)} \quad \text { (by Lemma 3.2). }
\end{aligned}
$$

And, whenever $(f ; \pi) \in H_{k}$ is such that $g_{\nu}(f ; \pi)=1$ for all $1 \leq \nu \leq c(\pi)$, we have

$$
\widetilde{\theta_{i}^{k}}(f ; \pi)=\prod_{\nu=1}^{c(\pi)} \theta_{i}\left(g_{\nu}(f ; \pi)\right)=\prod_{\nu=1}^{c(\pi)} 1=1 .
$$

Hence

$$
\begin{aligned}
A_{i, r} & =\frac{1}{(p-1)^{k} \cdot k !} \sum_{\pi \in \mathfrak{S}_{k}} \sum_{\substack{f \in H^{k} \text { such that } \\
g_{\nu}(f ; \pi)=1 \text { for all } 1 \leq \nu \leq c(\pi)}}(p-1)^{c(\pi)} \\
& =\frac{1}{(p-1)^{k} \cdot k !} \sum_{\pi \in \mathfrak{S}_{k}}(p-1)^{c(\pi)} \cdot|\mathcal{G}(\pi)|,
\end{aligned}
$$

where, for any $\pi \in \mathfrak{S}_{k}, \mathcal{G}(\pi)=\left\{f \in H^{k} \mid g_{\nu}(f ; \pi)=1\right.$ for all $\left.1 \leq \nu \leq c(\pi)\right\}$.

Now, if we write $\pi=\pi_{1} * \cdots * \pi_{c(\pi)}$, a product of disjoint cycles, we see that $f \in \mathcal{G}(\pi)$ if and only if, after reordering the "coordinates" of $f$ according to the cycles of $\pi, f$ is of the form $\left(f_{1}, \ldots, f_{c(\pi)}\right)$, where each $f_{\nu}$ is a $\left|\pi_{\nu}\right|$-tuple of elements of (the abelian group) $H$ whose product is $g_{\nu}(f, \pi)=1$. So, for each $1 \leq \nu \leq c(\pi)$, we can choose the first $\left|\pi_{\nu}\right|-1$ coordinates of $f_{\nu}$ to be anything we want in $H$, and the last coordinate is imposed by the condition $g_{\nu}(f, \pi)=1$. This means that, for each $1 \leq \nu \leq c(\pi)$, we have $(p-1)^{\left|\pi_{\nu}\right|-1}$ choices for $f_{\nu}$, so that

$$
|\mathcal{G}(\pi)|=\prod_{\nu=1}^{c(\pi)}(p-1)^{\left|\pi_{\nu}\right|-1} .
$$

We therefore have

$$
\begin{aligned}
(p-1)^{k} \cdot k ! \cdot A_{i, r} & =\sum_{\pi \in \mathfrak{S}_{k}}(p-1)^{c(\pi)} \cdot \prod_{\nu=1}^{c(\pi)}(p-1)^{\left|\pi_{\nu}\right|-1}=\sum_{\pi \in \mathfrak{S}_{k}} \prod_{\nu=1}^{c(\pi)}(p-1)^{\left|\pi_{\nu}\right|} \\
& =\sum_{\pi \in \mathfrak{S}_{k}}(p-1)^{\sum_{\nu=1}^{c(\pi)}\left|\pi_{\nu}\right|}=\sum_{\pi \in \mathfrak{S}_{k}}(p-1)^{k}=(p-1)^{k} \cdot k !
\end{aligned}
$$

and $A_{i, r}=1$, as claimed.

Note that our argument shows that, for any $\pi \in \mathfrak{S}_{k}$,

$$
\sum_{f \in H^{k}} \widetilde{\psi_{r}^{k}}(f ; \pi) \cdot \widetilde{\widetilde{\theta}_{i}^{k}(f ; \pi)}=(p-1)^{k}
$$

We will use this to prove the next result.

Lemma 4.1 can be extended as follows: 
Lemma 4.2. For any $i \in \sim^{\prime} I$ and any partitions $\alpha$ and $\beta$ of $k$, the multiplicity of the irreducible character $\widetilde{\theta_{i}^{k}} \otimes \zeta_{\alpha}$ in $\operatorname{Res}_{H_{k}}^{G_{k}}\left(\widetilde{\psi_{r}^{k}} \otimes \varphi_{\beta}\right)$ is $\delta_{\alpha, \beta}$.

Proof. Take any $i \in I$ and any partitions $\alpha$ and $\beta$ of $k$, and let $B_{i, r, \alpha, \beta}$ be the multiplicity of $\widetilde{\theta_{i}^{k}} \otimes \zeta_{\alpha}$ in $\operatorname{Res}_{H_{k}}^{G_{k}}\left(\widetilde{\psi_{r}^{k}} \otimes \varphi_{\beta}\right)$. Then

$$
\begin{aligned}
& B_{i, r, \alpha, \beta}=\frac{1}{\left|H_{k}\right|} \sum_{(f ; \pi) \in H_{k}} \widetilde{\psi_{r}^{k}}(f ; \pi) \cdot \widetilde{\widetilde{\theta}_{i}^{k}(f ; \pi)} \cdot \varphi_{\beta}(f ; \pi) \cdot \overline{\zeta_{\alpha}(f ; \pi)} \\
& =\frac{1}{\left|H_{k}\right|} \sum_{\pi \in \mathfrak{S}_{k}} \chi_{\beta}(\pi) \overline{\chi_{\alpha}(\pi)} \cdot\left(\sum_{f \in H^{k}} \widetilde{\psi_{r}^{k}}(f ; \pi) \cdot \widetilde{\widetilde{\theta}_{i}^{k}(f ; \pi)}\right) \\
& =\frac{1}{\left|H_{k}\right|} \sum_{\pi \in \mathfrak{S}_{k}} \chi_{\beta}(\pi) \overline{\chi_{\alpha}(\pi)} \cdot(p-1)^{k} \quad(\text { by }(5)) \\
& =\frac{(p-1)^{k}}{\left|H^{k}\right|} \cdot \frac{1}{\left|\mathfrak{S}_{k}\right|} \sum_{\pi \in \mathfrak{S}_{k}} \chi_{\beta}(\pi) \overline{\chi_{\alpha}(\pi)} \\
& =\left\langle\chi_{\beta}, \chi_{\alpha}\right\rangle_{\mathfrak{S}_{k}} \\
& =\delta_{\alpha, \beta} \text {, }
\end{aligned}
$$

as claimed.

To prove our next result on multiplicities, we will use the following, which is an easy corollary of Mackey's Theorem (see [2, Theorem (5.6)]) and Frobenius Reciprocity.

Theorem 4.3 (Mackey). Let $\mathcal{K}, \mathcal{H} \leq \mathcal{G}$ be finite groups, and $x_{1}, \ldots, x_{m}$ be representatives for the $(\mathcal{H}, \mathcal{K})$-double cosets in $\mathcal{G}$ (i.e. $\left.\mathcal{G}=\mathcal{H} x_{1} \mathcal{K} \dot{\cup} \cdots \dot{\mathcal{H}} x_{m} \mathcal{K}\right)$. Then, for any class functions $S$ and $T$ of $\mathcal{H}$ and $\mathcal{K}$ respectively, we have

$$
\left\langle\operatorname{Ind}_{\mathcal{H}}^{\mathcal{G}}(S), \operatorname{Ind}_{\mathcal{K}}^{\mathcal{G}}(T)\right\rangle_{\mathcal{G}}=\sum_{i=1}^{m}\left\langle\operatorname{Res}_{\mathcal{H}^{x_{i}} \mathcal{H}}^{\mathcal{H}^{x_{i}}}\left(S^{x_{i}}\right), \operatorname{Res}_{\mathcal{H}^{x_{i}} \cap \mathcal{K}}^{\mathcal{K}}(T)\right\rangle_{\mathcal{H}^{x_{i}} \cap \mathcal{K}}
$$

where the class function $S^{x_{i}}$ of $\mathcal{H}^{x_{i}}=x_{i}^{-1} \mathcal{H} x_{i}$ is defined by $S^{x_{i}}(u)=S\left(x_{i} u x_{i}^{-1}\right)$ for all $u \in \mathcal{H}^{x_{i}}(1 \leq i \leq m)$.

We can now prove the following

Theorem 4.4. For any $i \in I$, any $0 \leq j \leq k$, and any $\alpha \vdash k, \beta \vdash j$ and $\gamma \vdash k-j$, we have

$$
\left\langle\operatorname{Ind}_{H_{k}}^{G_{k}}\left(\widetilde{\theta_{i}^{k}} \otimes \zeta_{\alpha}\right), \operatorname{Ind}_{G_{j} \times G_{k-j}}^{G_{k}}\left(\left(\widetilde{\psi_{r}^{j}} \otimes \varphi_{\beta}\right) \otimes\left(\widetilde{\psi_{i}^{k-j}} \otimes \varphi_{\gamma}\right)\right)\right\rangle_{G_{k}}=c_{\beta, \gamma}^{\alpha},
$$

the Littlewood-Richardson coefficient for the symmetric group $\mathfrak{S}_{k}$ (see [3, Theorem 2.8.13]).

Remark: in the above statement, and in the rest of the paper, we denote some tensor products by $\otimes$ instead of $\otimes$ to emphasize the fact that they are outer tensor products.

Proof. We start by noticing that, if $j=0$ or $j=k$, then $G_{j} \times G_{k-j}=G_{k}$. For any $\alpha, \beta, \gamma \vdash k$, we let, in a natural way, $c_{\emptyset, \gamma}^{\alpha}=\delta_{\alpha, \gamma}$ and $c_{\beta, \emptyset}^{\alpha}=\delta_{\alpha, \beta}$. Then, if $j=0$, 
the claim becomes

$$
\left\langle\operatorname{Ind}_{H_{k}}^{G_{k}}\left(\widetilde{\theta_{i}^{k}} \otimes \zeta_{\alpha}\right), \widetilde{\psi_{i}^{k}} \otimes \varphi_{\gamma}\right\rangle_{G_{k}}=c_{\emptyset, \gamma}^{\alpha}=\delta_{\alpha, \gamma}
$$

which is true for any $\alpha, \gamma \vdash k$ by Theorem 3.3 and Frobenius Reciprocity. If, on the other hand, $j=k$, then the claim becomes

$$
\left\langle\operatorname{Ind}_{H_{k}}^{G_{k}}\left(\widetilde{\theta_{i}^{k}} \otimes \zeta_{\alpha}\right), \widetilde{\psi_{r}^{j}} \otimes \varphi_{\beta}\right\rangle_{G_{k}}=c_{\beta, \emptyset}^{\alpha}=\delta_{\alpha, \beta},
$$

which is true for any $\alpha, \beta \vdash k$ by Lemma 4.2 and Frobenius Reciprocity.

From now on, we therefore fix any $0<j<k$. Take any $\alpha \vdash k, \beta \vdash j$ and $\gamma \vdash k-j$, and let

$$
C_{i, j, \alpha, \beta, \gamma}=\left\langle\operatorname{Ind}_{H_{k}}^{G_{k}}\left(\widetilde{\theta_{i}^{k}} \otimes \zeta_{\alpha}\right), \operatorname{Ind}_{G_{j} \times G_{k-j}}^{G_{k}}\left(\left(\widetilde{\psi_{r}^{j}} \otimes \varphi_{\beta}\right) \otimes\left(\widetilde{\psi_{i}^{k-j}} \otimes \varphi_{\gamma}\right)\right)\right\rangle_{G_{k}} .
$$

We will apply Theorem 4.3 to the groups $\mathcal{G}=G_{k}, \mathcal{H}=H_{k}$ and $\mathcal{K}=G_{j} \times G_{k-j}$. Since $H_{k}$ contains (a copy of) $\mathfrak{S}_{k}$, which itself contains representatives for the left cosets of $G_{j} \times G_{k-j}$ in $G_{k}$ (which are the same as representatives for the left cosets of $\mathfrak{S}_{j} \times \mathfrak{S}_{k-j}$ in $\left.\mathfrak{S}_{k}\right)$, there is a single $\left(H_{k}, G_{j} \times G_{k-j}\right)$-double coset in $G_{k}$. Thus we have $G_{k}=H_{k} \cdot\left(G_{j} \times G_{k-j}\right)$ and, with the notations of Theorem $4.3, m=1$ and $x_{1}=1$. Also, $H_{k}^{1} \cap\left(G_{j} \times G_{k-j}\right)=H_{k} \cap\left(G_{j} \times G_{k-j}\right)=H_{j} \times H_{k-j}$. Hence, by Theorem 4.3, we have

$\left.C_{i, j, \alpha, \beta, \gamma}=\left\langle\operatorname{Res}_{H_{j} \times H_{k-j}}^{H_{k}}\left(\widetilde{\theta_{i}^{k}} \otimes \zeta_{\alpha}\right), \operatorname{Res}_{H_{j} \times H_{k-j}}^{G_{j} \times G_{k-j}}\left(\widetilde{\left(\psi_{r}^{j}\right.} \otimes \varphi_{\beta}\right) \otimes\left(\widetilde{\psi_{i}^{k-j}} \otimes \varphi_{\gamma}\right)\right)\right\rangle_{H_{j} \times H_{k-j}}$.

Now, for any $(f ; \pi) \in H_{j} \times H_{k-j}$, we can write $(f ; \pi)=\left(f^{(j)} ; \pi^{(j)}\right) \otimes\left(f^{(k-j)} ; \pi^{(k-j)}\right)$ in such a way that

$$
\begin{aligned}
\widetilde{\theta_{i}^{k}}(f ; \pi) & =\prod_{\nu=1}^{c(\pi)} \theta_{i}\left(g_{\nu}(f ; \pi)\right) \\
& =\prod_{\nu=1}^{c\left(\pi^{(j)}\right)} \theta_{i}\left(g_{\nu}\left(f^{(j)} ; \pi^{(j)}\right)\right) \cdot \prod_{\nu=1}^{c\left(\pi^{(k-j)}\right)} \theta_{i}\left(g_{\nu}\left(f^{(k-j)} ; \pi^{(k-j)}\right)\right) \\
& =\widetilde{\theta}_{i}^{j}\left(f^{(j)} ; \pi^{(j)}\right) \cdot \widetilde{\theta_{i}^{k-j}}\left(f^{(k-j)} ; \pi^{(k-j)}\right)
\end{aligned}
$$

(we only have to be careful, when seeing $\pi=\pi^{(j)} * \pi^{(k-j)}$ as an element of $\mathfrak{S}_{j} \times \mathfrak{S}_{k-j}$, that $\mathfrak{S}_{j}$ and $\mathfrak{S}_{k-j}$ do act on the indices we want).

This shows that

$$
\operatorname{Res}_{H_{j} \times H_{k-j}}^{H_{k}}\left(\widetilde{\theta_{i}^{k}}\right)=\widetilde{\theta_{i}^{j}} \otimes \widetilde{\theta_{i}^{k-j}} .
$$

Now, by the Littlewood-Richardson Rule (see [3, Theorem 2.8.13]), we have

$$
\operatorname{Res}_{\mathfrak{S}_{j} \times \mathfrak{S}_{k-j}}^{\mathfrak{S}_{k}}\left(\chi_{\alpha}\right)=\sum_{\substack{\mu \vdash j \\ \nu \vdash k-j}} c_{\mu, \nu}^{\alpha} \cdot \chi_{\mu} \otimes \chi_{\nu} .
$$

Since $\left\langle\operatorname{Res}_{H_{j} \times H_{j-k}}^{H_{k}}\left(\zeta_{\alpha}\right), \zeta_{\mu} \otimes \zeta_{\nu}\right\rangle_{H_{j} \times H_{k-j}}=\left\langle\operatorname{Res}_{\mathfrak{S}_{j} \times \mathfrak{S}_{k-j}}^{\mathfrak{S}_{k}}\left(\chi_{\alpha}\right), \chi_{\mu} \otimes \chi_{\nu}\right\rangle_{\mathfrak{S}_{j} \times \mathfrak{S}_{k-j}}$ and $\zeta_{\alpha}(1)=\chi_{\alpha}(1)$, this easily gives

$$
\operatorname{Res}_{H_{j} \times H_{j-k}}^{H_{k}}\left(\zeta_{\alpha}\right)=\sum_{\substack{\mu \vdash j \\ \nu \vdash k-j}} c_{\mu, \nu}^{\alpha} \cdot \zeta_{\mu} \otimes \zeta_{\nu} .
$$


Using (6) and (8), we obtain

$$
\operatorname{Res}_{H_{j} \times H_{k-j}}^{H_{k}}\left(\widetilde{\theta_{i}^{k}} \otimes \zeta_{\alpha}\right)=\sum_{\substack{\mu \vdash j \\ \nu \vdash k-j}} c_{\mu, \nu}^{\alpha} \cdot\left(\widetilde{\theta_{i}^{j}} \otimes \zeta_{\mu}\right) \otimes\left(\widetilde{\theta_{i}^{k-j}} \otimes \zeta_{\nu}\right) .
$$

We also have

(10) $\left.\left.\operatorname{Res}_{H_{j} \times H_{k-j}}^{G_{j} \times G_{k-j}}\left(\widetilde{\left(\psi_{r}^{j}\right.} \otimes \varphi_{\beta}\right) \otimes\left(\widetilde{\psi_{i}^{k-j}} \otimes \varphi_{\gamma}\right)\right)=\operatorname{Res}_{H_{j}}^{G_{j}} \widetilde{\psi_{r}^{j}} \otimes \varphi_{\beta}\right) \otimes \operatorname{Res}_{H_{k-j}}^{G_{k-j}}\left(\widetilde{\psi_{i}^{k-j}} \otimes \varphi_{\gamma}\right)$.

Together, (9) and (10) yield that $C_{i, j, \alpha, \beta, \gamma}$ is equal to

$$
\left.\left\langle\sum_{\substack{\mu \vdash j \\ \nu \vdash k-j}} c_{\mu, \nu}^{\alpha} \cdot\left(\widetilde{\theta_{i}^{j}} \otimes \zeta_{\mu}\right) \otimes\left(\widetilde{\theta_{i}^{k-j}} \otimes \zeta_{\nu}\right), \operatorname{Res}_{H_{j}}^{G_{j}} \widetilde{\psi_{r}^{j}} \otimes \varphi_{\beta}\right) \otimes \operatorname{Res}_{H_{k-j}}^{G_{k-j}}\left(\widetilde{\psi_{i}^{k-j}} \otimes \varphi_{\gamma}\right)\right\rangle_{H_{j} \times H_{k-j}},
$$

which in turn is the same as

$$
\left.\left.\left.\sum_{\substack{\mu \vdash j \\ \nu \vdash k-j}} c_{\mu, \nu}^{\alpha} \cdot\left\langle\widetilde{\theta_{i}^{j}} \otimes \zeta_{\mu}, \operatorname{Res}_{H_{j}}^{G_{j}} \widetilde{\left(\psi_{r}^{j}\right.} \otimes \varphi_{\beta}\right)\right\rangle_{H_{j}} \cdot \widetilde{\theta_{i}^{k-j}} \otimes \zeta_{\nu}, \operatorname{Res}_{H_{k-j}}^{G_{k-j}} \widetilde{\left(\psi_{i}^{k-j}\right.} \otimes \varphi_{\gamma}\right)\right\rangle_{H_{k-j}} .
$$

Now, by Lemma 4.2, we have, for all $\mu \vdash j$,

$$
\left.\left\langle\widetilde{\theta_{i}^{j}} \otimes \zeta_{\mu}, \operatorname{Res}_{H_{j}}^{G_{j}} \widetilde{\left(\psi_{r}^{j}\right.} \otimes \varphi_{\beta}\right)\right\rangle_{H_{j}}=\delta_{\mu, \beta}
$$

Also, by Lemma 3.1, we have

$$
\operatorname{Res}_{H_{k-j}}^{G_{k-j}}\left(\widetilde{\psi_{i}^{k-j}} \otimes \varphi_{\gamma}\right)=\operatorname{Res}_{H_{k-j}}^{G_{k-j}}\left(\widetilde{\psi_{i}^{k-j}}\right) \otimes \operatorname{Res}_{H_{k-j}}^{G_{k-j}}\left(\varphi_{\gamma}\right)=\widetilde{\theta_{i}^{k-j}} \otimes \zeta_{\gamma} .
$$

Hence, for all $\nu \vdash k-j$, we have

$$
\left.\left\langle\widetilde{\theta_{i}^{k-j}} \otimes \zeta_{\nu}, \operatorname{Res}_{H_{k-j}}^{G_{k-j}} \widetilde{\psi_{i}^{k-j}} \otimes \varphi_{\gamma}\right)\right\rangle_{H_{k-j}}=\left\langle\widetilde{\theta_{i}^{k-j}} \otimes \zeta_{\nu}, \widetilde{\theta_{i}^{k-j}} \otimes \zeta_{\gamma}\right\rangle_{H_{k-j}}=\delta_{\nu, \gamma}
$$

(since both are irreducible characters of $H_{k-j}$, the same if and only if $\nu=\gamma$ ).

Finally, using (11), (12) and (13), we obtain

$$
C_{i, j, \alpha, \beta, \gamma}=\sum_{\substack{\mu \vdash j \\ \nu \vdash k-j}} c_{\mu, \nu}^{\alpha} \cdot \delta_{\mu, \beta} \cdot \delta_{\nu, \gamma}=c_{\beta, \gamma}^{\alpha},
$$

as claimed.

We can now finally state and prove the main result of this section.

Theorem 4.5. For any $i \in I$, any integer $k \geq 1$ and $\alpha \vdash k$, we have

$$
\left.\operatorname{Ind}_{H_{k}}^{G_{k}}\left(\widetilde{\theta_{i}^{k}} \otimes \zeta_{\alpha}\right)=\sum_{j=0}^{k} \sum_{\substack{\beta \vdash j \\ \gamma \vdash k-j}} c_{\beta, \gamma}^{\alpha} \cdot \operatorname{Ind}_{G_{j} \times G_{k-j}}^{G_{k}}\left(\widetilde{\left(\psi_{r}^{j}\right.} \otimes \varphi_{\beta}\right) \otimes\left(\widetilde{\psi_{i}^{k-j}} \otimes \varphi_{\gamma}\right)\right) .
$$

Proof. Since the characters appearing on the right-hand side are pairwise distinct irreducible characters of $G_{k}$, Theorem 4.4 shows that all we have to prove is that the left-hand side and right-hand side characters have the same degree.

For the left-hand side, we easily see that

$$
\operatorname{Ind}_{H_{k}}^{G_{k}}\left(\widetilde{\theta_{i}^{k}} \otimes \zeta_{\alpha}\right)(1)=\left[G_{k}: H_{k}\right] \cdot\left(\widetilde{\theta_{i}^{k}} \otimes \zeta_{\alpha}\right)(1)=\left[G_{k}: H_{k}\right] \cdot \zeta_{\alpha}(1)=p^{k} \cdot \chi_{\alpha}(1) .
$$


Now, for any $0 \leq j \leq k$ and any $\beta \vdash j$ and $\gamma \vdash k-j$, we have

$$
\begin{aligned}
& \left.\Delta_{j, \beta, \gamma}:=\operatorname{Ind}_{G_{j} \times G_{k-j}}^{G_{k}}\left(\widetilde{\left(\psi_{r}^{j}\right.} \otimes \varphi_{\beta}\right) \otimes\left(\widetilde{\psi_{i}^{k-j}} \otimes \varphi_{\gamma}\right)\right)(1) \\
& =\left[G_{k}: G_{j} \times G_{k-j}\right] \cdot \widetilde{\psi_{r}^{j}}(1) \cdot \varphi_{\beta}(1) \cdot \widetilde{\psi_{i}^{k-j}}(1) \cdot \varphi_{\gamma}(1) \\
& =\frac{k !}{j ! \cdot(k-j) !} \cdot\left(\psi_{r}(1)\right)^{j} \cdot \varphi_{\beta}(1) \cdot \varphi_{\gamma}(1) \\
& =\left(\begin{array}{c}
k \\
j
\end{array}\right) \cdot(p-1)^{j} \cdot \chi_{\beta}(1) \cdot \chi_{\gamma}(1) .
\end{aligned}
$$

We therefore obtain, for the right-hand side,

$$
\begin{aligned}
\Delta_{k} & \left.:=\sum_{j=0}^{k} \sum_{\substack{\beta \vdash j \\
\gamma \vdash k-j}} c_{\beta, \gamma}^{\alpha} \cdot \operatorname{Ind}_{G_{j} \times G_{k-j}}^{G_{k}}\left(\widetilde{\left(\psi_{r}^{j}\right.} \otimes \varphi_{\beta}\right) \otimes\left(\widetilde{\psi_{i}^{k-j}} \otimes \varphi_{\gamma}\right)\right) \\
& =\sum_{j=0}^{k} \sum_{\substack{\beta \vdash j \\
\gamma \vdash k-j}} c_{\beta, \gamma}^{\alpha} \cdot \Delta_{j, \beta, \gamma} \\
& =\sum_{j=0}^{k} \sum_{\substack{\beta \vdash j \\
\gamma \vdash k-j}} c_{\beta, \gamma}^{\alpha} \cdot\left(\begin{array}{c}
k \\
j
\end{array}\right) \cdot(p-1)^{j} \cdot \chi_{\beta}(1) \cdot \chi_{\gamma}(1) \\
& =\sum_{j=0}^{k}\left(\begin{array}{c}
k \\
j
\end{array}\right) \cdot(p-1)^{j} \cdot\left(\sum_{\substack{\beta \vdash j \\
\gamma \vdash k-j}} c_{\beta, \gamma}^{\alpha} \cdot \chi_{\beta}(1) \cdot \chi_{\gamma}(1)\right) \\
& =\left(\sum_{j=0}^{k}\left(\begin{array}{l}
k \\
j
\end{array}\right) \cdot(p-1)^{j}\right) \cdot \chi_{\alpha}(1) \quad \text { (by (7)) } \\
& =p^{k} \cdot \chi_{\alpha}(1)
\end{aligned}
$$

(since $\left.p^{k}=((p-1)+1)^{k}=\sum_{j=0}^{k}\left(\begin{array}{l}k \\ j\end{array}\right) \cdot(p-1)^{j}\right)$. This concludes the proof.

\section{MAIN RESUlT}

We can now state and prove our main result (Theorem 5.1). Take any positive integer $w$. Recall that the coefficients we wish to find are the multiplicities of the irreducible characters of $H_{w}$ in the restriction to $H_{w}$ of any irreducible character of $G_{w}$. Equivalently, we want to decompose into irreducibles of $G_{w}$ the induced character $\operatorname{Ind}_{H_{w}}^{G_{w}}(\xi)$ of any $\xi \in \operatorname{Irr}\left(H_{w}\right)$. Hence we take any $\alpha=\left(\alpha^{1}, \ldots, \alpha^{r-1}, \alpha^{r+1}, \ldots, \alpha^{p}\right) \Vdash w$, and consider $\xi^{\alpha} \in \operatorname{Irr}\left(H_{w}\right)$. Recall that $\xi^{\alpha}$ is given by

$$
\left.\xi^{\alpha}=\operatorname{Ind}_{\prod_{i \in I}^{H_{w} H_{\left|\alpha^{i}\right|}}}^{H_{i \in I}} \widetilde{\theta_{i}^{\left|\alpha^{i}\right|}} \otimes \zeta_{\alpha^{i}}\right)
$$

or, letting $H_{\alpha}=\prod_{i \in I} H_{\left|\alpha^{i}\right|}$ and $\Theta^{\alpha}=\prod_{i \in I} \widetilde{\theta_{i}^{\left|\alpha^{i}\right|}} \otimes \zeta_{\alpha^{i}}$, by $\xi^{\alpha}=\operatorname{Ind}_{H_{\alpha}}^{H_{w}}\left(\Theta^{\alpha}\right)$.

The diagram below explains our strategy. 


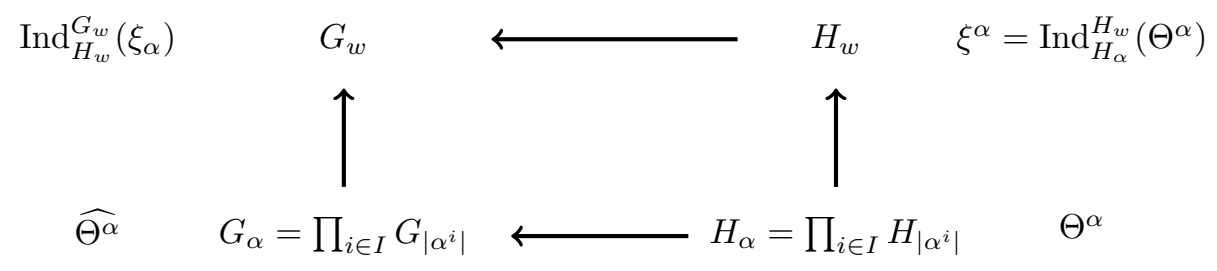

Starting from $\Theta^{\alpha} \in \operatorname{Irr}\left(H_{\alpha}\right)$, instead of going right around the above diagram to compute $\operatorname{Ind}_{H_{w}}^{G_{w}}\left(\operatorname{Ind}_{H_{\alpha}}^{H_{w}}\left(\Theta^{\alpha}\right)\right)$, we will go left to compute (the same character) $\operatorname{Ind}_{G_{\alpha}}^{G_{w}}\left(\operatorname{Ind}_{H_{\alpha}}^{G_{\alpha}}\left(\Theta^{\alpha}\right)\right)$. Now, since $H_{\left|\alpha^{i}\right|} \leq G_{\left|\alpha^{i}\right|}$ for all $i \in I$, we have

$$
\widehat{\Theta^{\alpha}}:=\operatorname{Ind}_{H_{\alpha}}^{G_{\alpha}}\left(\Theta^{\alpha}\right)=\operatorname{Ind}_{\prod_{i \in I} H_{\left|\alpha^{i}\right|}}^{\prod_{i \in I} G_{\left|\alpha^{i}\right|}}\left(\prod_{i \in I} \widetilde{\theta_{i}^{\left|\alpha^{i}\right|}} \otimes \zeta_{\alpha^{i}}\right)=\prod_{i \in I} \operatorname{Ind}_{H_{\left|\alpha^{i}\right|}}^{G_{\left|\alpha^{i}\right|}}\left(\widetilde{\theta_{i}^{\left|\alpha^{i}\right|}} \otimes \zeta_{\alpha^{i}}\right) .
$$

By Theorem 4.5, we therefore obtain

$$
\begin{aligned}
& \widehat{\Theta^{\alpha}}=\prod_{i \in I} \operatorname{Ind}_{H_{\left|\alpha^{i}\right|}}^{G_{\left|\alpha^{i}\right|}}\left(\widetilde{\theta_{i}^{\left|\alpha^{i}\right|}} \otimes \zeta_{\alpha^{i}}\right) \\
& \left.=\prod_{i \in I} \sum_{j=0}^{\left|\alpha^{i}\right|} \sum_{\substack{\beta \vdash j \\
\gamma \vdash\left|\alpha^{i}\right|-j}} c_{\beta, \gamma}^{\alpha^{i}} \cdot \operatorname{Ind}_{G_{j} \times G_{\left|\alpha^{i}\right|-j}}^{G_{1} \alpha^{i} \mid}\left(\widetilde{\left(\widetilde{\psi_{r}^{j}}\right.} \otimes \varphi_{\beta}\right) \otimes\left(\widetilde{\psi_{i}^{\left|\alpha^{i}\right|-j}} \otimes \varphi_{\gamma}\right)\right) \\
& \left.=\sum_{\substack{0 \leq j_{i} \leq\left|\alpha^{i}\right| \\
(i \in I)}} \sum_{\substack{\beta^{i} \vdash j_{i} \\
\gamma^{i} \vdash \mid \alpha \in I \\
(i \in I)}} \prod_{i \in I} c_{\beta^{i}, \gamma^{i}}^{\alpha^{i}} \cdot \operatorname{Ind}_{G_{j_{i}} \times G_{\left|\alpha^{i}\right|-j_{i}}}^{G_{\mid} \alpha^{i} \mid}\left(\widetilde{\psi_{r}^{j_{i}}} \otimes \varphi_{\beta^{i}}\right) \otimes\left(\widetilde{\psi_{i}^{\left|\alpha^{i}\right|-j_{i}}} \otimes \varphi_{\gamma^{i}}\right)\right) \\
& =: \quad \sum_{\substack{\left.0 \leq j_{i} \leq\left|\alpha^{i}\right| \\
i \in I\right)}} \sum_{\substack{\beta^{i} \vdash j_{i} \\
\gamma^{i} \vdash\left|j^{i}\right|-j_{i} \\
(i \in I)}}\left(\prod_{i \in I} c_{\beta^{i}, \gamma^{i}}^{\alpha^{i}}\right) \cdot \widehat{\Theta_{\alpha, \beta, \gamma}},
\end{aligned}
$$

where we have

$$
\begin{aligned}
& \left.\widehat{\Theta_{\alpha, \beta, \gamma}}=\prod_{i \in I} \operatorname{Ind}_{G_{j_{i}} \times G_{\left|\alpha^{i}\right|-j_{i}} \alpha^{i} \mid}\left(\widetilde{\psi_{r}^{j_{i}}} \otimes \varphi_{\beta^{i}}\right) \otimes\left(\widetilde{\psi_{i}^{\left|\alpha^{i}\right|-j_{i}}} \otimes \varphi_{\gamma^{i}}\right)\right) \\
& =\operatorname{Ind} \prod_{\prod_{i \in I} G_{i} G_{j_{i}} \times G_{\left|\alpha^{i}\right|-j_{i}}}\left(\prod_{i \in I}\left(\widetilde{\psi_{r}^{j_{i}}} \otimes \varphi_{\beta^{i}}\right) \otimes\left(\widetilde{\psi_{i}^{\left|\alpha^{i}\right|-j_{i}}} \otimes \varphi_{\gamma^{i}}\right)\right) .
\end{aligned}
$$

Inducing to $G_{w}$, we now have

$$
\operatorname{Ind}_{H_{w}}^{G_{w}}\left(\xi_{\alpha}\right)=\operatorname{Ind}_{G_{\alpha}}^{G_{w}}\left(\widehat{\Theta^{\alpha}}\right)=\sum_{\substack{\left.0 \leq j_{i} \leq\left|\alpha^{i}\right| \\ i \in I\right)}} \sum_{\substack{\beta^{i} \vdash j_{i} \\ \gamma^{i} \vdash\left|\alpha^{i}\right|-j_{i} \\(i \in I)}}\left(\prod_{i \in I} c_{\beta^{i}, \gamma^{i}}^{\alpha^{i}}\right) \operatorname{Ind}_{G_{\alpha}}^{G_{w}}\left(\widehat{\Theta_{\alpha, \beta, \gamma}}\right)
$$


where, if we write $J=\left(j_{1}, \ldots, j_{r-1}, j_{r+1}, \ldots, j_{p}\right),|J|=\sum_{i \in I} j_{i}$ and $\alpha-J=$ $\left(\alpha^{1}-j_{1}, \ldots, \alpha^{r-1}-j_{r-1}, \alpha^{r+1}-j_{r+1}, \ldots, \alpha^{p}-j_{p}\right)$, we have

$$
\begin{aligned}
& \left.\operatorname{Ind}_{G_{\alpha}}^{G_{w}}\left(\widetilde{\Theta_{\alpha, \beta, \gamma}}\right)=\operatorname{Ind}_{\prod_{i \in I}^{G_{w}} G_{j_{i}} \times G_{\left|\alpha^{i}\right|-j_{i}}}\left(\prod_{i \in I} \widetilde{\left(\psi_{r}^{j_{i}}\right.} \otimes \varphi_{\beta^{i}}\right) \otimes\left(\widetilde{\psi_{i}^{\left|\alpha^{i}\right|-j_{i}}} \otimes \varphi_{\gamma^{i}}\right)\right) \\
& \left.=\operatorname{Ind}_{\prod_{i \in I}^{G_{w}} G_{j_{i}} \times \prod_{i \in I} G_{\left|\alpha^{i}\right|-j_{i}}}\left(\prod_{i \in I} \widetilde{\psi_{r}^{j_{i}}} \otimes \varphi_{\beta^{i}}\right) \otimes \prod_{i \in I}\left(\widetilde{\psi_{i}^{\left|\alpha^{i}\right|-j_{i}}} \otimes \varphi_{\gamma^{i}}\right)\right) \\
& =\operatorname{Ind}_{G_{J} \times G_{\alpha-J}}^{G_{w}}\left(\widetilde{\prod_{i \in I}}\left(\widetilde{\psi_{r}^{j_{i}}} \otimes \varphi_{\beta^{i}}\right) \otimes \prod_{i \in I}\left(\widetilde{\psi_{i}^{\left|\alpha^{i}\right|-j_{i}}} \otimes \varphi_{\gamma^{i}}\right)\right) \\
& =\operatorname{Ind}_{G_{|J|} \times G_{\alpha-J}}^{G_{w}}\left(\operatorname{Ind}_{G_{J} \times G_{\alpha-J}}^{G_{|J|} \times G_{\alpha-J}}\left(\widetilde{\prod_{i \in I}}\left(\widetilde{\psi_{r}^{j_{i}}} \otimes \varphi_{\beta^{i}}\right) \otimes \prod_{i \in I}\left(\widetilde{\psi_{i}^{\left|\alpha^{i}\right|-j_{i}}} \otimes \varphi_{\gamma^{i}}\right)\right)\right) \\
& \left.=\operatorname{Ind}_{G_{|J|} \times G_{\alpha-J}}^{G_{w}}\left(\operatorname{Ind}_{G_{J}}^{G_{|J|}}\left(\prod_{i \in I} \widetilde{\left(\psi_{r}^{j_{i}}\right.} \otimes \varphi_{\beta^{i}}\right)\right) \otimes \prod_{i \in I}\left(\widetilde{\psi_{i}^{\left|\alpha^{i}\right|-j_{i}}} \otimes \varphi_{\gamma^{i}}\right)\right) .
\end{aligned}
$$

And, iterating [4, Proposition 4.1], we have

$$
\left.\left.\operatorname{Ind}_{G_{J}}^{G_{|J|}}\left(\prod_{i \in I} \widetilde{\psi_{r}^{j_{i}}} \otimes \varphi_{\beta^{i}}\right)\right)=\sum_{\gamma^{r} \vdash|J|} c_{\left(\beta^{i}, i \in I\right)}^{\gamma^{r}} \cdot \widetilde{\left(\psi_{r}^{|J|}\right.} \otimes \varphi_{\gamma^{r}}\right),
$$

where $c_{\left(\beta^{i}, i \in I\right)}^{\gamma^{r}}=\left\langle\chi_{\gamma^{r}}, \operatorname{Ind}_{\prod_{i \in I} \mathfrak{S}_{j_{i}}}^{\mathfrak{S}_{|J|}}\left(\prod_{i \in I} \chi_{\beta^{i}}\right)\right\rangle_{\mathfrak{S}_{|J|}}$ is the coefficient obtained by iterating the Littlewood-Richardson Rule.

We therefore obtain

$$
\begin{aligned}
& \left.\operatorname{Ind}_{G_{\alpha}}^{G_{w}}\left(\widehat{\Theta_{\alpha, \beta, \gamma}}\right)=\sum_{\gamma^{r} \vdash|J|} c_{\left(\beta^{i}, i \in I\right)}^{\gamma^{r}} \cdot \operatorname{Ind}_{G_{|J|} \times G_{\alpha-J}}^{G_{w}}\left(\widetilde{\psi_{r}^{|J|}} \otimes \varphi_{\gamma^{r}}\right) \otimes \prod_{i \in I}\left(\widetilde{\psi_{i}^{\left|\alpha^{i}\right|-j_{i}}} \otimes \varphi_{\gamma^{i}}\right)\right) \\
& =\sum_{\gamma^{r} \vdash|J|} c_{\left(\beta^{i}, i \in I\right)}^{\gamma^{r}} \cdot \operatorname{Ind}_{G_{\left|\gamma^{r}\right| \times \prod_{i \in I}}^{G_{w}} G_{\left|\gamma^{i}\right|}}\left(\widetilde{\left.\left(\widetilde{\psi_{r}^{\left|\gamma^{r \mid}\right|}} \otimes \varphi_{\gamma^{r}}\right) \otimes \prod_{i \in I}\left(\widetilde{\psi_{i}^{\left|\gamma^{i}\right|}} \otimes \varphi_{\gamma^{i}}\right)\right),}\right.
\end{aligned}
$$

whence

$$
\operatorname{Ind}_{G_{\alpha}}^{G_{w}}\left(\widehat{\Theta_{\alpha, \beta, \gamma}}\right)=\sum_{\gamma^{r} \vdash|J|} c_{\left(\beta^{i}, i \in I\right)}^{\gamma^{r}} \cdot \chi^{\left(\gamma^{1}, \ldots, \gamma^{p}\right)} .
$$

Finally, this yields

$$
\operatorname{Ind}_{H_{w}}^{G_{w}}\left(\xi^{\alpha}\right)=\sum_{\substack{0 \leq j_{i} \leq\left|\alpha^{i}\right| \\(i \in I)}} \sum_{\substack{\beta^{i} \vdash j_{i} \\ \gamma^{i} \mid \alpha^{i} i-j_{i} \\(i \in I)}} \sum_{\gamma^{r} \vdash \sum_{i \in I} j_{i}}\left(\prod_{i \in I} c_{\beta^{i}, \gamma^{i}}^{\alpha^{i}}\right) \cdot c_{\left(\beta^{i}, i \in I\right)}^{\gamma^{r}} \cdot \chi^{\left(\gamma^{1}, \ldots, \gamma^{p}\right)}
$$

which is the decomposition of $\operatorname{Ind}_{H_{w}}^{G_{w}}\left(\xi^{\alpha}\right)$ into irreducible characters of $G_{w}$. Note that each irreducible $\chi^{\left(\gamma^{1}, \ldots, \gamma^{p}\right)}$ appears (with multiplicity) several times, corresponding to the choice of the $\beta^{i}$,s $(i \in I)$. This can now be rewritten as

Theorem 5.1. For any integer $w>0$ and $\alpha=\left(\alpha^{1}, \ldots, \alpha^{r-1}, \alpha^{r+1}, \ldots, \alpha^{p}\right) \Vdash w$, we have

$$
\operatorname{Ind}_{H_{w}}^{G_{w}}\left(\xi^{\alpha}\right)=\sum_{\gamma=\left(\gamma^{1}, \ldots, \gamma^{p}\right) \Vdash w} k_{\alpha, \gamma} \cdot \chi^{\gamma}
$$


where, for any $\gamma=\left(\gamma^{1}, \ldots, \gamma^{p}\right) \Vdash w$,

$$
k_{\alpha, \gamma}=\sum_{\substack{\beta^{i} \vdash\left|\alpha^{i}\right|-\left|\gamma^{i}\right| \\(i \in I)}}\left(\prod_{i \in I} c_{\beta^{i}, \gamma^{i}}^{\alpha^{i}}\right) \cdot c_{\left(\beta^{i}, i \in I\right)}^{\gamma^{r}} .
$$

In particular, $k_{\alpha, \gamma}=0$ unless $\left|\gamma^{i}\right| \leq\left|\alpha^{i}\right|$ for all $i \in I$.

Remark 5.2. We recover from Theorem 5.1 the fact that our basic set $\left\{\chi^{\gamma} \in\right.$ $\left.\operatorname{Irr}\left(G_{w}\right) \mid \gamma^{r}=\emptyset\right\}$ corresponds to $\operatorname{Irr}\left(H_{w}\right)$ (see Theorem 3.3 and Remark 3.4). Indeed, for $\xi^{\alpha}$ to appear in $\operatorname{Res}_{H_{w}}^{G_{w}}\left(\chi^{\gamma}\right)$, we must have $\left|\alpha^{i}\right| \geq\left|\gamma^{i}\right|$ for all $i \in I$. But, if $\gamma^{r}=\emptyset$, then we already have $\sum_{i \in I}\left|\gamma_{i}\right|=\sum_{i=1}^{p}\left|\gamma_{i}\right|=w=\sum_{i \in I}\left|\alpha_{i}\right|$, so that we can only have $\left|\alpha^{i}\right|=\left|\gamma^{i}\right|$ for all $i \in I$. We then get $k_{\alpha, \gamma}=\left(\prod_{i \in I} c_{\emptyset, \gamma^{i}}^{\alpha^{i}}\right) \cdot c_{(\emptyset, \ldots, \emptyset)}^{\emptyset}=\prod_{i \in I} \delta_{\alpha^{i}, \gamma^{i}}$, so that $k_{\alpha, \gamma}=\delta_{\hat{\alpha}, \gamma}$ (with the notation of Theorem 3.3).

\section{REFERENCES}

[1] O. Brunat and J. Gramain. A basic set for the alternating group. J. Reine Angew. Math., 641:177-202, 2010.

[2] I. Isaacs. Character theory of finite groups. AMS Chelsea Publishing, Providence, RI, 2006. Corrected reprint of the 1976 original [Academic Press, New York; MR0460423].

[3] G. James and A. Kerber. The representation theory of the symmetric group, volume 16 of Encyclopedia of Mathematics and its Applications. Addison-Wesley Publishing Co., Reading, Mass., 1981.

[4] I. Stein. The Littlewood-Richardson rule for wreath products with symmetric groups and the quiver of the category $F$ ? $\mathbf{F I}_{n}$. Comm. Algebra, 45(5):2105-2126, 2017.

Institute of Mathematics, University of Aberdeen, King's College, Fraser Noble Bullding, Aberdeen AB24 3UE, UK

E-mail address: jbgramain@abdn.ac.uk

National University of Singapore, Department of Mathematics, Faculty of Science, Level 4, Block S17, 10 Lower Kent Ridge Road, Singapore 119076, Singapore.

E-mail address: matmae@nus.edu.sg 